\title{
A smart energy consumption manager based on protues simulation
}

Mohammed Abdulhussein Mohammed, Alaa Desher Farhood, Ahmed Jabbar Abid

Technical Electronics Department, Technical Instructors Training Institute, Middle Technical University, Iraq

\begin{tabular}{ll}
\hline \hline Article Info & ABSTRACT \\
\cline { 3 - 3 } Article history: & $\begin{array}{l}\text { In the proposed system, a strategy for the control of energy consumption by } \\
\text { home appliances is provided. The statistics of previous energy production } \\
\text { and consumption of a case study city are used in providing the strategy. In } \\
\text { Received Apr 8, 2019 } \\
\text { the design of the proposed system, home appliances are categorized into } \\
\text { Accepted Jul 22, 2019 }\end{array}$ \\
$\begin{array}{l}\text { three levels of priority, even though it can be more than three. In this article } \\
\text { the control of energy consumption is achieved using a real time energy } \\
\text { consumption manager (ECM) based on stored data without the need for real } \\
\text { time communication. The system which is proposed here is affordable and } \\
\text { simple. Also, it does not require grid upgrade or power line communication } \\
\text { through the grid. }\end{array}$
\end{tabular}

management

Load curve

Microcontroller

Power grid

This is an open access article under the CC BY-SA license.

\section{Corresponding Author:}

Mohammed Abdulhussein Mohammed,

Technical Electronics Dept., Technical Instructors Training Institute,

Middle Technical University,

Baghdad, Iraq.

Email: dradnanmtu@gmail.com

\section{INTRODUCTION}

In order to measure the adequacy of generating energy, margins of installed capacity over peak-load is usually used. A decrease in these margins has been recorded in many IEA countries, but are still highly present in some areas [1]. In majority of third world countries, the power production for supply of houses and other facilities within a given period of time is insufficient. This is because some of these countries try to reduce the cost of power production or emission of $\mathrm{Co} 2$ through different strategies which include less power production. Others countries or cities that try to rely on only renewable energy encounter some challenges that are associated with abrupt weather changes and other conditions.

Global efforts have been directed towards promoting the use of clean energy for the protection of the environment, and these efforts have also involved the scheduling of energy consumption pattern so as to meet the increasing demand for power $[2,3]$. One of the challenges confronting business and households is the inadequate supply of power. One of the strategies used in addressing the issue of insufficient power within cities is power supply scheduling. With this scheduling, the public overuses their time slot when the power is supplied to them. This in turn has made the extension of the time of power supply difficult [4].

Against this backdrop, a solution which can be used in individual households is offered in this research. This solution permits smart control and monitoring of temporary load-shedding of the individual house unit's power consumption anytime the given limit of power consumption is exceeded [5, 6]. The specified power limit can be made flexible, such that it can be reduced or increased depending on the availability of the total power allocated to a given area at particular times of the day or year. 
Unlike the smart grid, the designed system is capable of regulating energy consumption without requiring a power line communication. More so, the system possesses numerous features like low cost, simplicity, easy development and installation because it is based on programmable microcontroller, and it is possible to update saved data by overwriting the external RAM or by sending an SMS if the GSM optional modem connected $[7,8]$.

This paper concentrates on the electric energy engineering and the power industry departments, the electricity corporation and power grid enterprises that are the subjects of social power saving in addition to scientific and rational power consumption, and demand-side management (DSM) implementation. They play important roles in sponsoring the enhancement of overall efficiency for the social power consumption and the development of DSM work.

\section{LITERATURE SURVEY}

In a system that whereby the enterprise of power production is separated from that of distribution, the distribution enterprise becomes totally responsible for the implementation of Demand-Side Management (DSM) because large scale electric consumers are directly involved [9, 10]. In the proposed system, it is assumed that the energy which is produced is insufficient, and there is need for the demand side to reduce the load for a given period of time during the year [11]. In some studies, the researchers proposed a strategy for energy control that is pricing-based; their proposed strategies are aimed at eliminating the peak load for smart grid. Based on the price, energy consumers are able to regulate the energy which they consume so as to make a tradeoff between the reduction of load and electricity cost [12].

Other researchers have recommended that demand side management in smart grid can be implemented by the scheduling of techniques used for home appliance [13, 14]. The power system has been immensely affected by consumers' growing demand, thereby posing some challenges to the power generation system in terms of quantity and quality of power supply [15]. This problem in smart grid can be solved through efficient consumption and economical generation because it is combined with information communication technologies. Smart grid provides the opportunities for the use of various pricing schemes that are capable of increasing efficiency of scheduling techniques [16, 17].

In spite of the issues and challenges discussed in [18], a more suitable solution could be smart grid. In [19], a number of the issues and challenges associated with smart grid were presented alongside their solutions. Several researchers have suggested that the use of a load management application system that is based on smart grid privacy policy for energy management service, be employed within an environment of energy management service [20]. In order to enable robustness and load balancing, the authors during study [21] proposed the use of cloud computing and data centers.

\section{CASE STUDY}

The case study used in this study is a city with an annual load curve that is presented in Figure 1, and a day load curve in Figure 2 [22]. The annual curve is a representation of an inadequate power generation from the month of May to September; here, the reduction of the power demand below the values in the curve is required. Some countries increase the cost of KW-H unit with the aim of reducing the load [16]. For instance, in countries like Iraq, the load is decreased by shutting down the supply in some cities, but this strategy has an adverse effect on the quality of life [23]. A Similar procedure is used through the day load peak. However, in the proposed system, a novel strategy is presented as seen in the flowchart contained in Figure 3, in which, the house appliances are divided into three different levels according to priorities [24, 25].

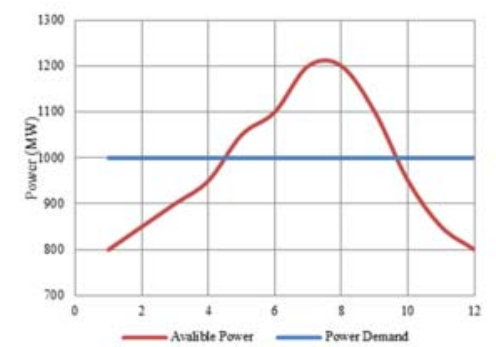

Figure 1. Annual load curve

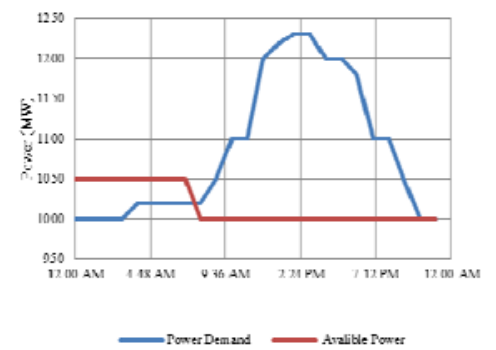

Figure 2. A day load curve

Int J Pow Elec \& Dri Syst Vol. 11, No. 1, Mar 2020 : $143-150$ 


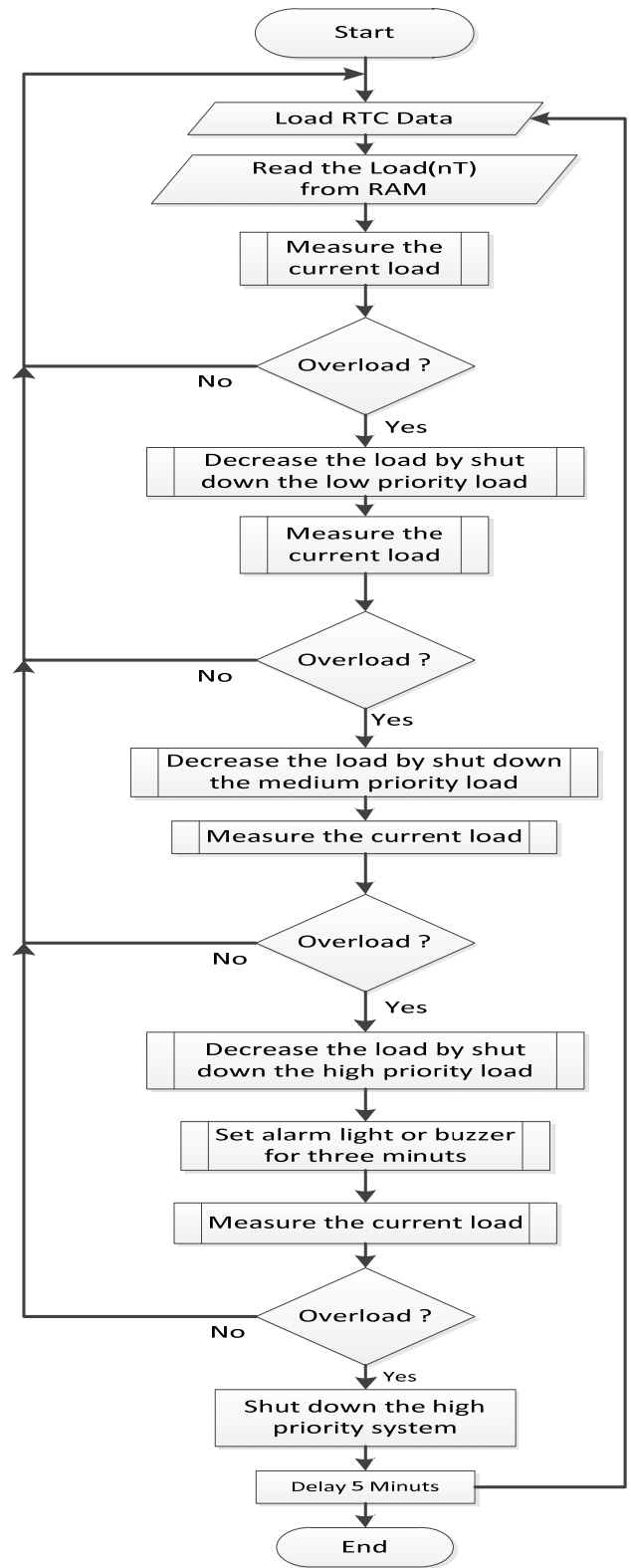

Figure 3. Flowchart for the proposed software

\section{SYSTEM HARDWARE CIRCUITRY}

The ATmega32 microcontroller is the major part of the proposed design, and it is responsible for the management of every peripheral by means of the different kinds of communication protocols. Figure 4, shows that communication takes place between the microcontroller and GSM modem through RS232 (TX and RX)@9600 bps. This occurs while the microcontroller is in communication with a real time controller (RTC) and the external RAM through Inner-integrated protocol (I2C). It is also managed by a $4 \times 4$ matrix keypad through 8 pins, and drives the LCD display in 4 bits mode. An 8-ADC is possessed by the microcontroller, thereby being able to measure the real time AC current so as to read the Current Transformer (CT) conditioning signal.

\subsection{ATmega32 Microcontroller}

The microcontroller (ATmega32) which is used in this study, possesses all the peripheral attributes which are needed by design. Some of the attributes include compare mode, one 16-bit Timer/Counter with separate prescaler, real time counter having a separate oscillator, capture mode, 8-channel, four PWM

A smart energy consumption manager based on protues simulation (Mohammed Abdulhussein Mohammed) 
channels, 10-bit ADC, programmable serial USART, byte-oriented two-wire serial interface, master/slave SPI serial interface and a watchdog timer that is programmable and having a separate on-chip oscillator. The connection between the peripherals and the microcontroller is presented in Figure 5 while the configurations of the signal line are shown in Table 1.

Table 1. Signal Line Configurations

\begin{tabular}{lll}
\hline Used Labels & Pin No. & Descriptions \\
\hline RST & 9 & Connected to push button to reset the system. \\
X1-X2 & $13-12$ & Crystal (20MHz) \\
CT & 40 & Current Transformer Conditioner signal \\
$1-4$ & $33-36$ & 4x4 Keypad \\
A-D & $18-21$ & \\
D4-D7, RS,MOSI & $1-6$ & LCD Screen, MOSI as EN signal \\
SCL & 22 & I $^{2}$ s serial clock \\
SDA & 23 & I $^{2}$ C serial data \\
& & For external RAM \& RTC \\
High & 24 & High priority relay enables \\
Medium & 25 & Medium priority relay enables \\
Low & 26 & Low priority relay enables \\
RX & 14 & RS232 received data \\
TX & 15 & RS232 transmitted data \\
& & For GSM Modem \\
MOSI, MISO, SCK & $6,7,8$ & For programming purposes ICSP1 \\
\hline
\end{tabular}

\subsection{Load manager}

Through three layers, the facility is supplied with the main power. The load is controlled by three relays depending on the various levels of priorities.

\subsection{Real time controller (RTC)}

It is the responsibility to manage the time over the day and date over the year. The RTC, which was used is the RTC (DS1307) that possesses a serial real-time clock (RTC) with low power, full binary-coded decimal (BCD) clock/calendar plus 56 bytes of NV SRAM. With this, the transfer of data is done serially through an $\mathrm{I} 2 \mathrm{C}$ bidirectional bus.

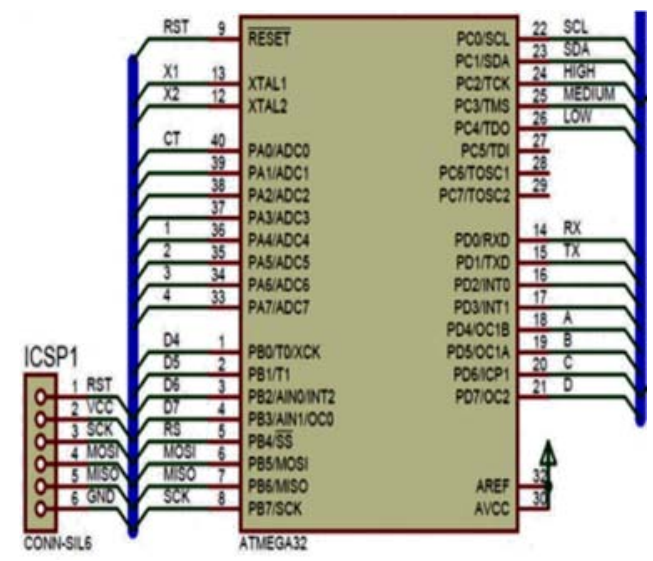

Figure 4. System Block Diagram

Figure 5. Microcontroller Pins Connections

The kind of information provided by the clock/calendar includes information on hours, second, minutes, day, date, month and year, provides seconds, minutes, hours, day, date, month, and year information. In the case of months with less than 31 days, an automatic adjustment is made to the end of the month date, including corrections for leap year. The format used by the clock is either, the 24-hour or 12hour format. The DS1307 is capable of detecting power failure and automatically switching to backup supply 
through its built-in power-sense circuit. The operation of time-keeping remains while the part operates from the backup supply.

\subsection{External RAM}

Based on the design, the load is a matter of time Load (nT), where $n$ denotes the number of the sample per annum and $\mathrm{T}$ represents the time interval between samples. The design of the system allows it to save as sample hourly throughout the year, therefore making $n=365 \times 24=8760$ and $\mathrm{T}=1$ hour "it could be more or less as a matter of accuracy". The format in which all the samples will be saved is "XX. XX" A. This format saved in two bytes which make the RAM > 17520 Bytes or equal to $32 \mathrm{~KB}$.

The use of 24FC512 was employed by the system because it has good characteristics such as low power CMOS technology, a single supply with operation down to $1.7 \mathrm{v}, 2$-Wire Serial Interface, I2C $\mathrm{C}^{\mathrm{TM}}$ compatible, allows flow for up to eight devices, $100 \mathrm{kHz}$ and $400 \mathrm{kHz}$ Clock Compatibility, and Schmitt trigger inputs suppress noise.

\subsection{Display}

To display the allowed load and instant load, an LCD display $(2 \times 16)$ characters were used. The LCD connection as well as the two rows of displaying data is shown inFigure 6 . In the first row the load demand in ampere (ID) is displayed, while the second row is a display of the maximum allowed current at this moment.

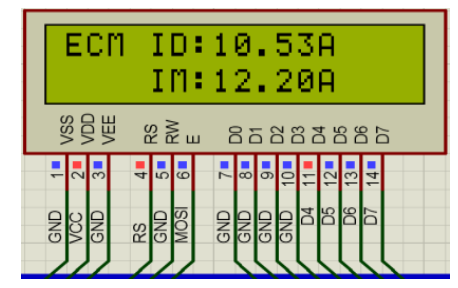

Figure 6. LCD Screen (2x16 characters)

\subsection{Transformer less Power Supply}

The power supply which adopted by the design is that requires no transformer that makes use of a parallel RC for the reduction of the voltage through 1A fuse. Subsequent to this stage is the process of rectification using bridge, and afterwards regulated at $5 \mathrm{vdc}$.

\subsection{Current transformer and conditioning}

As seen in Fig (7, the alternating electric currents are measured using the current transformer (CT). Current transformers, together with voltage (or potential) transformers (VT or PT), are known as instrument transformers. In an instance whereby the circuit current is extremely high for direct application on the instruments of measurement, a reduced current which is accurately equal to the circuit current is produced by the current transformer; this can be suitably connected to the measuring and recording instruments. In a current transformer, the instruments of measurement are separated from what may tagged as extremely high voltage in the monitored circuit. The use of current transformers is employed in protective relays and metering in the electrical power industry.

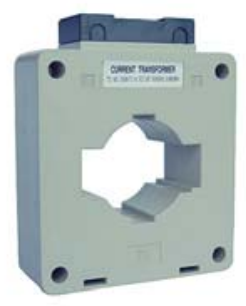

Figure 7. Current Transformer

A smart energy consumption manager based on protues simulation (Mohammed Abdulhussein Mohammed) 
In the proposed system, a CT with transfer ratio of (100-1) was utilized, so the maximum output current $\mathrm{I}_{\text {out }}$ will be $1 \mathrm{~A}$ if the input current $\mathrm{I}_{\text {in }}$ equal $100 \mathrm{~A}$ based on the (1).

$$
I_{\text {in }} * N_{p}=I_{\text {out }} * N_{s}
$$

In order to change the current to voltage and scale up the value of the current, the CT is terminated using a $3.3 \Omega / 1 \mathrm{~W}$ resistor. At this point, it is safe and the ratio of conversion value is $3.3 \mathrm{~V}$ to each $100 \mathrm{~A}$. The resistor is being paralleled by a Zener diode $5.1 \mathrm{~V}$ for the purpose of over voltage protection.

\subsection{Load Table of Priorities}

In an event whereby the produced power is inadequate, the load can be managed if the different levels of priority are considered by the system. Here, the demand load of the system is checked periodically to know if the allowed load has been exceeded, and then the appliances of low priority are shut down. The system checks again and if the load still exceeds, the appliances of medium priority will be shut down. More so, if the produced power is incapable of covering the high priority load, an alarm is raised by the system or an SMS is sent to the house owner to manually reduce the load, if not, the system will shut down in the entire house for three minutes, and afterwards, turn on the high priority so as to check again and this procedure will be repeated thrice and then stop until the produced power is able to cover the demand load. In the proposed system, the demand of the house owner is memorized by the system, and then it turns on other devices when power is available. Even though, the levels of priority can be determined by the user, the Table 2 shows the different levels of priority.

Table 2. List of Load Priorities

\begin{tabular}{ll}
\hline Priority Level & \multicolumn{1}{c}{ Appliances } \\
\hline High Level & $\begin{array}{l}\text { Security System, Smoke alarm } \\
\text { Emergency light and all the other important } \\
\text { appliances }\end{array}$ \\
Medium Level & $\begin{array}{l}\text { Air conditioning system Refrigerators } \\
\text { Water heater, etc. } \\
\text { Low level }\end{array}$ \\
& It could be any appliance that not affected by losing \\
& power.
\end{tabular}

\section{RESULT AND DISCUSSION}

The use of Protues software was used in the system simulation; all the components that were used were simulated including the microcontroller. The simulated circuit was divided into various areas as seen in Figure 18. Keypad which is optional was only plugged in during the phase of initialization. In Figure 9 the two-layer system printed circuit board (PCB) is presented. The proposed system is cost effective.

The amount spent on all the components of the system is given in Table 3 . The cost of the prototype is $\approx 52$ USD, which can be lesser when purchased in large quantity for mass production. The cost for the $\mathrm{PCB}$ and installation of components is $\approx 38$ USD. Therefore, the total cost is $\approx 90$ USD. Although, the use of the keypad and GSM modem is optional, they cost $\approx 35$ USD. Lastly, the plotting of the PCB is done in 3D mode as presented in Figure 10, which is dependent on minimum size. In summary, the proposed system requires no smart grid communication, it is portable, cost efficient, easy to install, lightweight and easy development because it is based on an integrated circuit that is programmable (MCU), GSM based and dynamic data about power availability.

Int J Pow Elec \& Dri Syst Vol. 11, No. 1, Mar 2020 : 143 - 150 


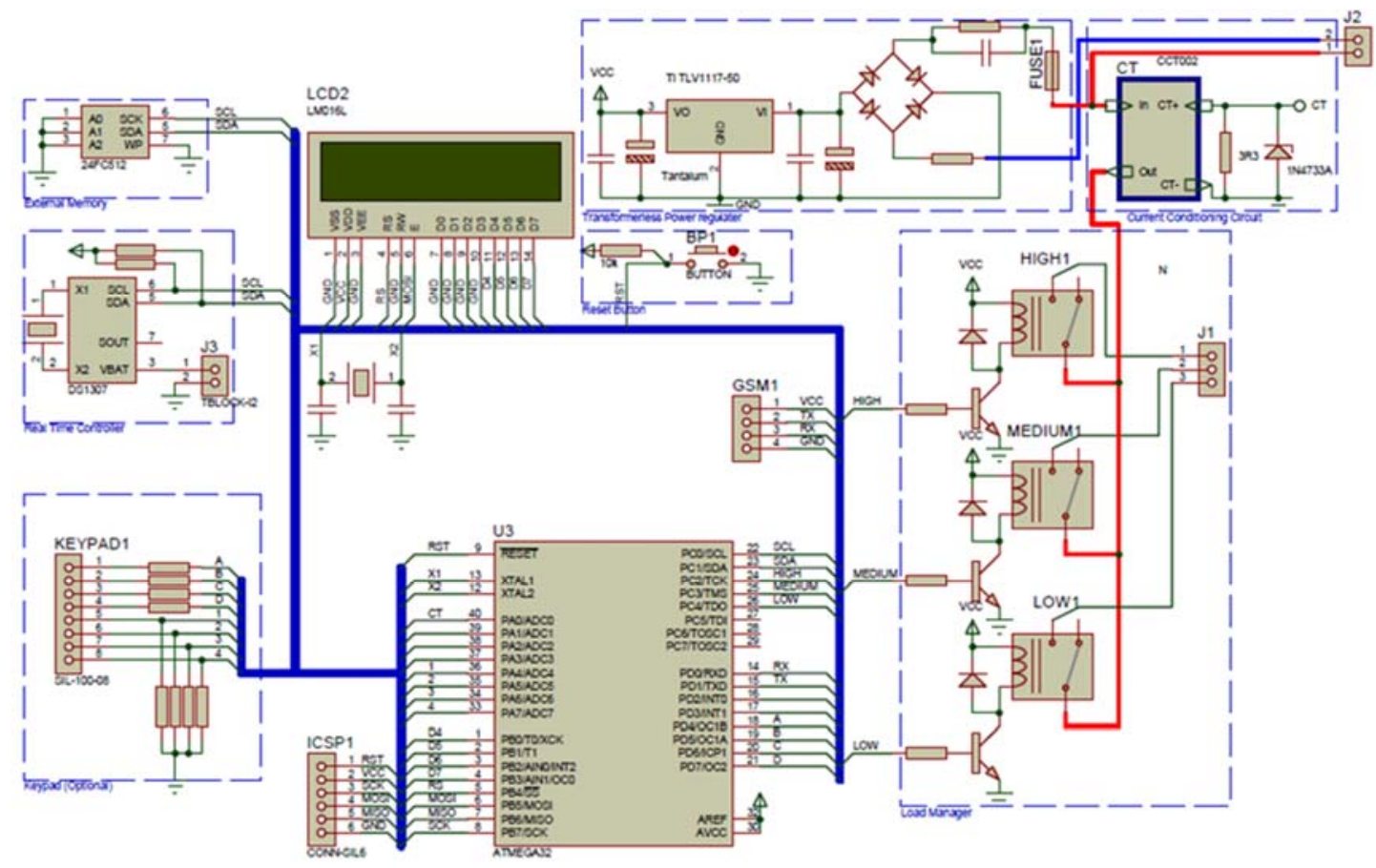

Figure 1. System hardware circuitry

Table 3. Bill of Materials

\begin{tabular}{lcc}
\hline QTY & References & Value \\
\hline 7 & C1-C2,C3, C4, C5,C6, C7 & $22 \mathrm{p}, 10 \mathrm{uF}, 0.1 \mathrm{uF}$ \\
$1 \mathrm{uF}, 2 \mathrm{u} 2,470 \mathrm{u}$ \\
17 & $\mathrm{R} 2, \mathrm{R} 10-\mathrm{R} 11, \mathrm{R} 4 \mathrm{R} 6, \mathrm{R} 7-\mathrm{R} 9, \mathrm{R} 12, \mathrm{R} 13-\mathrm{R} 16$ & 4.7k,470R, 100k,330R, 470k, 100R, \\
& R17, R18, R19 & $3 \mathrm{R} 3$ \\
1 & $\mathrm{U} 1$ & $\mathrm{DS} 1307$ \\
1 & $\mathrm{U} 2$ & $24 \mathrm{FC} 512$ \\
1 & $\mathrm{U} 4$ & ATMEGA32 \\
1 & $\mathrm{U} 5$ & TI TLV1117-50 \\
3 & Q1-Q3 & BC547 \\
4 & D1-D3, D4 & 1N4148, 1N4733A \\
1 & BAT1 & $3 \mathrm{~V}$ \\
1 & BR1 & $2 \mathrm{~W} 04 \mathrm{G}$ \\
1 & FUSE & $1 \mathrm{~A}$ \\
3 & High, low,medium & G2R-14-AC120 Relay \\
1 & ICSP & CONN-SIL6 \\
2 & J1, J2 & TBLOCK-I3,I2 \\
1 & LCD1 & LM016L \\
1 & RST & Micro switch \\
2 & X1, X2 & 20 MHz, 32.768KHz \\
\hline
\end{tabular}

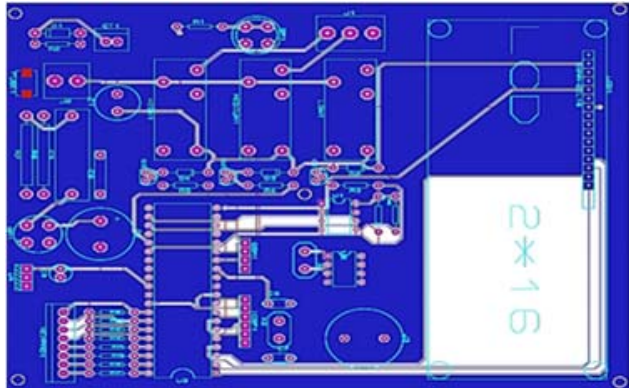

Figure 9. System PCB circuit

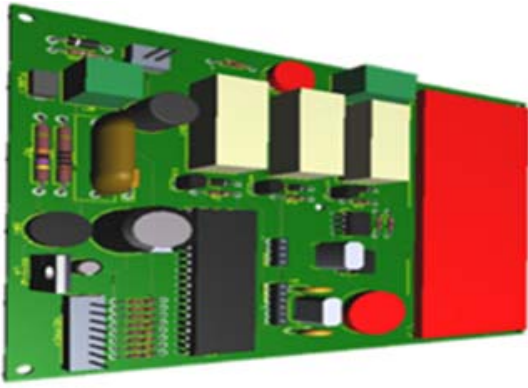

Figure 10. System 3D circuit 


\section{REFERENCES}

[1] François Nguyen and Ulrik Stridbaek, "Tackling Investment Challenges in Power Generation", In IEA Countries, Energy Market Experience. International Energy Agency (IEA), Head of Publications Service, 2007.

[2] Ali A. Abdulrazzaq, Adnan H. Ali, "Performance Investigation of Grid Connected Photovoltaic System Modelling Based on MATLAB Simulation", International Journal of Electrical and Computer Engineering (IJECE), Vol. 8 No. 6, 2018.

[3] Ahmed J. Abid, Fawzi M. Al-Naima, and Adnan H. Ali, "Comprehensive Modeling of PV Array based on Proteus Software", International Journal of Applied Engineering Research, Volume 13, Number, pp. 4440-4447, 2018.

[4] Ali A. Abdulrazzaq, Adnan H. Ali, "Efficiency Performances of Two MPPT Algorithms for PV System With Different Solar Panels Irradiances", International Journal of Power Electronics and Drive System (IJPEDS), Vol. 9 , No. 4, December 2018, pp. 1755 1764.

[5] Hussein A. Mohammed, Adnan H. Ali, "Effect of some Security Mechanisms on the Qos VoIP Application using OPNET", International Journal of Current Engineering and Technology, Vol.3, No.5, December, 2013.

[6] Hu Zhaoguang, Han Xinyang, and Wen Quan, "The Implementers of Demand-Side Management: Power Grid Enterprises," in Integrated Resource Strategic Planning and Power Demand-Side Management: Springer Berlin Heidelberg, 2013, pp. 219-286.

[7] Kye-Si Kwon, Wook-B. Kim, Byung-K. Min, Sung-J. Park, In-Ha Sung, Young S. Yoon, Kyung-Soo Lee, Jong-H. Lee, "Energy consumption reduction technology in manufacturing - A selective review of policies, standards, and research," International Journal of Precision Engineering and Manufacturing, vol. 10, no. 5, pp. 151-173, December 2009.

[8] Kai Ma, Guoqiang Hu, and C.J. Spanos, "Distributed Energy Consumption Control via Real-Time Pricing Feedback in Smart Grid," IEEE Transactions Control Systems Technology, vol. 22, no. 5, pp. 1907-1914, Sept. 2014.

[9] F. Al-Naima, R. Ali, and A. Abid, "Design of a control and data acquisition system for a multi-mode solar tracking farm," in European Workshop on Renewable Energy Systems, Antalya, Turkey, 2012.

[10] F. Al-Naima, R. Ali, A. Abid, Z. Ghassemlooy, and Z. Gao, "A New Power Line Communication Modem Design with Applications to Vast Solar Farm Management," in EPECS 2013, Istanbul, 2013.

[11] M.N. Ullah, N. Javaid, I. Khan, A. Mahmood, and M.U. Farooq, "Residential Energy Consumption Controlling Techniques to Enable Autonomous Demand Side Management in Future Smart Grid Communications," in Broadband and Wireless Computing, Communication and Applications (BWCCA), Compiègne, French, 28-30 Oct. 2013, pp. 545-550.

[12] Mohannad J. Mnati, Adnan H. Ali, Dimitar V. Bozalakov, Shahad Al-yousif, Alex Van den Bossche, "Design and Implementation of a Gate Driver Circuit for Three-Phase Grid Tide Photovoltaic Inverter Application", 7th International Conference on Renewable Energy Research and Applications (ICRERA), Paris, 14-17 Oct. 2018.

[13] Park Namje and Kim Marie, "Implementation of load management application system using smart grid privacy policy in energy management service environment," Cluster Computing, vol. 17, no. 3, pp. 653-664, Sep. 2014.

[14] Adnan H. Ali, Ali N. Abbas, M. H. Hassan," Performance Evaluation of IEEE802.11g WLANs Using OPNET Modeler", (AJER) Volume-02, Issue-12, pp-09-15.

[15] Fan Chun-I, Huang Shi-Yuan, and Artan William, "Design and implementation of privacy preserving billing protocol for smart grid," The Journal of Supercomputing, vol. 66, no. 2, pp. 841-862, November 2013.

[16] AJ Abid, AH Ali, "Smart monitoring of the consumption of home electrical energy", International Journal of Computer Trends and Technol., vol. 47, no. 2, pp. 142-148, , 2017.

[17] Adnan H. Ali, 2011, "Simultaneous measurements for tunable laser source linewidth with homodyne detection" Computer and Information Science, Vol. 4, No. 4; July 2011.

[18] MJ Mnati, A Hasan, Adnan H. Ali, DV Bozalakov, "Design and Implementation A Smart Monitoring and Controlling System of Three-Phase Photovoltaic Inverter Based on LoRa" - IOP Conference Series: Materials Science and Engineering 518 , 2nd International Conference on Sustainable Engineering Techniques 2019.

[19] Gabriele L, Salvatore C and Erica L "A Review of systems and technologies for smart homes and smart grids" Energies 9 pp 1-33, 2016.

[20] Adnan H. Ali, Farhood, A.D. "Design and Performance Analysis of the WDM Schemes for Radio over Fiber System with Different Fiber Propagation Losses", Fibers, vol, 7, 19, 2019.

[21] Giovanni P, Mario C, Antonio R and Jiahu Q, "Home energy management", Energies, vol, 10,(382), 2017.

[22] Farid K, Mohamed B, Yassine A and Abdelaziz K "Integrated energy management of a plug-in electric vehicle in residential distribution systems with renewables”, IEEE 24th International Symposium on Industrial Electronics (ISIE), pp 717-22, 2015.

[23] M. M. Kareem, M. Ismail, N. Arsad, M. F. Mansor, and A. H. Ali, 'Grid Based Clustering Technique in Wireless Sensor Network using Hierarchical Routing Protocol', in 2018 IEEE 4th International Symposium on Telecommunication Technologies (ISTT), pp. 1-5, 2018.

[24] Kaur, K.; Kaur, K. A study of power management techniques for Internet of Things (IoT). In Proceedings of the International Conference on Electrical, Electronics, and Optimization Techniques (ICEEOT), Chennai, India, 3-5; pp. 1781-1785, March 2016.

[25] Zhang, D.; Li, S.; Sun, M.; O’Neill, Z. An Optimal and Learning-Based Demand Response and Home Energy Management System. IEEE Trans. Smart Grid, 7, 1790-1801, 2016.

Int J Pow Elec \& Dri Syst Vol. 11, No. 1, Mar 2020 : 143 - 150 